\title{
Stratification of fire smoke and testing aerosol in a road tunnel: computer simulation
}

\author{
Peter Weisenpacher ${ }^{1, *}$, Jan Glasa ${ }^{1}$, and Lukas Valasek ${ }^{1}$ \\ ${ }^{1}$ Institute of Informatics, Slovak Academy of Sciences, Dubravska cesta 9, 84507 Bratislava, Slovakia
}

\begin{abstract}
The damage of the road tunnel during tunnel ventilation tests is prevented by using specific testing aerosol representing fire smoke. This study compares optical density profiles of aerosol and fire smoke using the well-known FDS simulation system, testing the suitability of aerosol to represent fire smoke realistically. The movement of aerosol and fire smoke downstream of the aerosol/fire source is investigated for a case of a $240 \mathrm{~m}$ long section of a road tunnel. Similarity of optical density profile for both cases under given conditions is confirmed. The influence of mesh resolution and the way of computational domain decomposition is evaluated as well. In case of fire smoke, the influence of domain decomposition and mesh resolution is very slight, while in case of aerosol it is more pronounced due to effect of relatively course numerical grid. The results support the hypothesis that the aerosol is suitable to represent the fire smoke for steady-state conditions occurring downstream of the fire in tunnel ventilation tests.
\end{abstract}

\section{Introduction}

Maintaining of smoke stratification is the primary task of ventilation strategy in case of fire in bi-directional road tunnels [1, 2, 3, 4]. Jet fans create prescribed target velocity of the flow maintaining stratification of smoke layer which spreads above the heads of people trapped both upstream and downstream of the fire. Tenable conditions for human life are thus preserved long enough to ensure safe evacuation. Proper functionality of ventilation is tested during ventilations tests of realistic fire scenarios required by authorities. Therefore, ventilation tests are an important part of fire safety measures.

However, it is impossible to perform full-scale ventilation tests using real fires in tunnels in operation. Fire would lead to the destruction of tunnel facilities as well as concrete tunnel walls. The problem is of special importance in Slovakia, where several highway road tunnels are under construction or have been planned [5, 6]. Therefore, a specific kind of testing aerosol [7] is used during tests instead of the smoke produced by fire. The aerosol is meant to visualize the smoke and must meet the following requirements:

- Optical density of aerosol is similar to that of considered fire smoke

- Heat produced during aerosol generation allows maintaining the aerosol stratification at ambient temperature

- Opacity sensors and smoke detectors respond correctly to the aerosol

- Heat does not damage the tunnel facilities

- Aerosol is not toxic and does not cause corrosion.
It is generated by a chemical reaction of 50 times lower heat release rate (HRR) than the HRR of the corresponding fire represented by aerosol. Optical density of the aerosol in a sufficiently long distance downstream of the modelled fire source should correspond to the optical density of the fire smoke. This way, devices installed in the tunnel are protected. Realistic smoke movement is achieved downstream of the modelled fire source and full scale fire and ventilation tests required by authorities can be conducted.

In [8] the movement of the aerosol in a road tunnel has been compared with the movement of the corresponding fire smoke by computer simulations using the $20 \mathrm{~cm}$ mesh resolution. Conditions under which both movements are similar were found. Proper mass production of the aerosol necessary to obtain similar optical density profiles for the aerosol and fire smoke were determined as well as the distance at which a steady-state movement of the smoke layer occurs.

The task has been solved by the well-known opensource code Fire Dynamics Simulator (FDS, version 6.5.2) $[9,10]$. The FDS is a CFD-based simulation system for modelling fire and fire-driven fluid flows. It solves numerically a form of conservation equations for low-speed, thermally-driven flows with an emphasis on the smoke and heat transport from fire.

In this study, more detailed research of smoke and aerosol stratification is performed. Simulations using finer mesh resolution are executed and its influence on simulation result is evaluated. Phenomena not captured by the $20 \mathrm{~cm}$ mesh resolution are analysed as well. Computational requirements of several simulations with

* Corresponding author: upsyweis@savba.sk 
finer mesh resolutions exceed the capabilities of personal computers. As our research demonstrated the applicability of parallel versions of FDS for tunnel fire simulations [11], parallel calculations are performed on the SIVVP high-performance computer (HPC) cluster at the Institute of Informatics, Slovak Academy of Sciences, Bratislava (Slovakia) [12]. Parallel MPI version 6.5.2 of the FDS is used and the influence of parallelism on simulations accuracy is also evaluated.

The results can increase the confidence on capability of the aerosol to represent the fire smoke during tunnel ventilations tests and improve the experiments settings.

\section{Fire Scenarios}

Geometrical model of $240 \mathrm{~m}$ long section of a road tunnel of horseshoe cross section was used for FDS simulations. The dimensions of the cross section of the tunnel (10.8 m width and $6.8 \mathrm{~m}$ height) are typical for road tunnels currently under construction in Slovakia $[13,14]$. The computational domain was identical with the tunnel dimensions: $240 \mathrm{~m} \mathrm{x} 10.8 \mathrm{~m}$ x $7.2 \mathrm{~m}$. Variable dynamic pressure was set on the left tunnel portal to obtain and stabilize a required target velocity of the air flow in the tunnel. The initial value of air flow velocity was $90 \%$ of the target velocity.

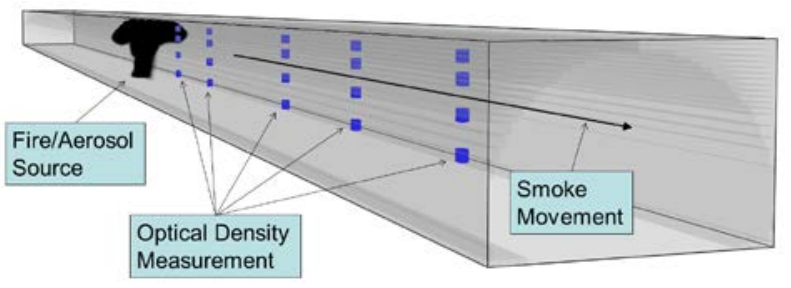

Fig. 1. Scheme of the tunnel.

$1 \mathrm{~m} . \mathrm{s}^{-1}$ target velocity typical for ventilation scenarios in bi-directional tunnels was used. The fire HRR was $1 \mathrm{MW}$ and the soot yield was 0.2. The fire source in fire scenario was modelled as a rectangular block located $10 \mathrm{~m}$ from the centre of the tunnel (i.e., $130 \mathrm{~m}$ from the left portal). Its top surface $\left(1 \mathrm{~m}^{2}\right)$ burned with the HRR per unit area (HRRPUA) of $1 \mathrm{MW} \cdot \mathrm{m}^{-2}$. Taking into account a low HRR of the chemical reaction generating the aerosol $(20 \mathrm{~kW})$, the source of aerosol in aerosol scenarios was modelled as a block with $0.2 \times 0.2$ $\mathrm{m}$ top surface (one mesh cell), which corresponds to the dimensions of real aerosol generators.

$1 \mathrm{MW}$ fire produced $5.0 \mathrm{~g}$ of soot per second. As buoyancy of the fire accelerated the spread of smoke under the tunnel ceiling in comparison with aerosol, a lower mass flux of aerosol was sufficient to create the same optical density profile as the fire smoke. In [8] the value of 2.7 g.s ${ }^{-1}$ for aerosol mass flow rate was determined as the most appropriate.

Five scenarios per fire and five per aerosol, respectively, were executed. Sequential scenarios using $20 \mathrm{~cm}$ mesh resolution were identical with the scenarios that have been used in [8] for fire and aerosol. Another set of sequential scenarios used refined mesh with 10 $\mathrm{cm}$. Due to extremely long wall-clock time and memory requirements, simulations with $5 \mathrm{~cm}$ mesh resolution (denoted $5 \mathrm{~cm}-8 \mathrm{M}$ ) were executed in parallel. The computational domain was decomposed into 8 meshes, each of them assigned to one MPI process (one CPU core). In order to evaluate the influence of parallelism on simulation accuracy, parallel calculations for 10 and 20 $\mathrm{cm}$ mesh resolution using 8 meshes were executed as well.

Optical density was evaluated by five quadruples of detectors at 6.0, 5.0, 3.4 and $1.6 \mathrm{~m}$ height at 25, 40, 65, 80 and $95 \mathrm{~m}$ from the fire/aerosol source. The values were used for evaluation of differences between scenarios.

The $200 \mathrm{~s}$ smoke movement was simulated using Intel Core i7-4790 CPU @ 3.60 GHz for sequential scenarios. For parallel scenarios SIVVP HPC cluster was used. It is an IBM dx360 M3 cluster consisting of 54 computational nodes (2x Intel E5645 @ 2.4 GHz CPU, 48 GB RAM); the total number of cores is 648. The nodes are connected by the Infiniband interconnection network with the bandwidth of $40 \mathrm{Gbit} / \mathrm{s}$ per link and direction.

The domain with $20 \mathrm{~cm}$ mesh resolution consists of $1200 \times 54 \times 36$ cells. The total number of cells is $2,332,800$. For the $10 \mathrm{~cm}$ and $5 \mathrm{~cm}$ mesh resolution, the total number of cells is 18,662,400 and 149,299,200, respectively. The wall-clock time strongly depends on specifications of each scenario, mainly on the mesh resolution. It is only 9 hours for $20 \mathrm{~cm}$ resolution; however; wall-clock time for $5 \mathrm{~cm}$ resolution scenario is more than 50 days.

\section{Simulation Results}

The qualitative results of the simulations are consistent with [8] (see Figures 2 and 3). Refined mesh resolution does not influence the main tendencies of smoke and aerosol spread. The most visible difference between the spread of fire smoke and aerosol, the back-layering occurring in the former case is beyond the scope of this study. Our analysis is focused on the steady-state movement of smoke being formed in a larger distance downstream. The smoke/aerosol layer looks very similar for all tested values of mesh resolution and division of computational domain in both fire and aerosol scenarios, respectively. However, several important differences can be determined and analysed.

The differences are evaluated using optical density values measured by particular detectors, averaged over the interval 150 - $200 \mathrm{~s}$, when steady-state movement of smoke/aerosol is achieved. This way, every scenario is characterized by 20 averaged values of optical density. The sum of squared differences between the timeaveraged optical density values measured by all detectors for the particular couple of scenarios is considered to be a measure of the differences between both scenarios. 

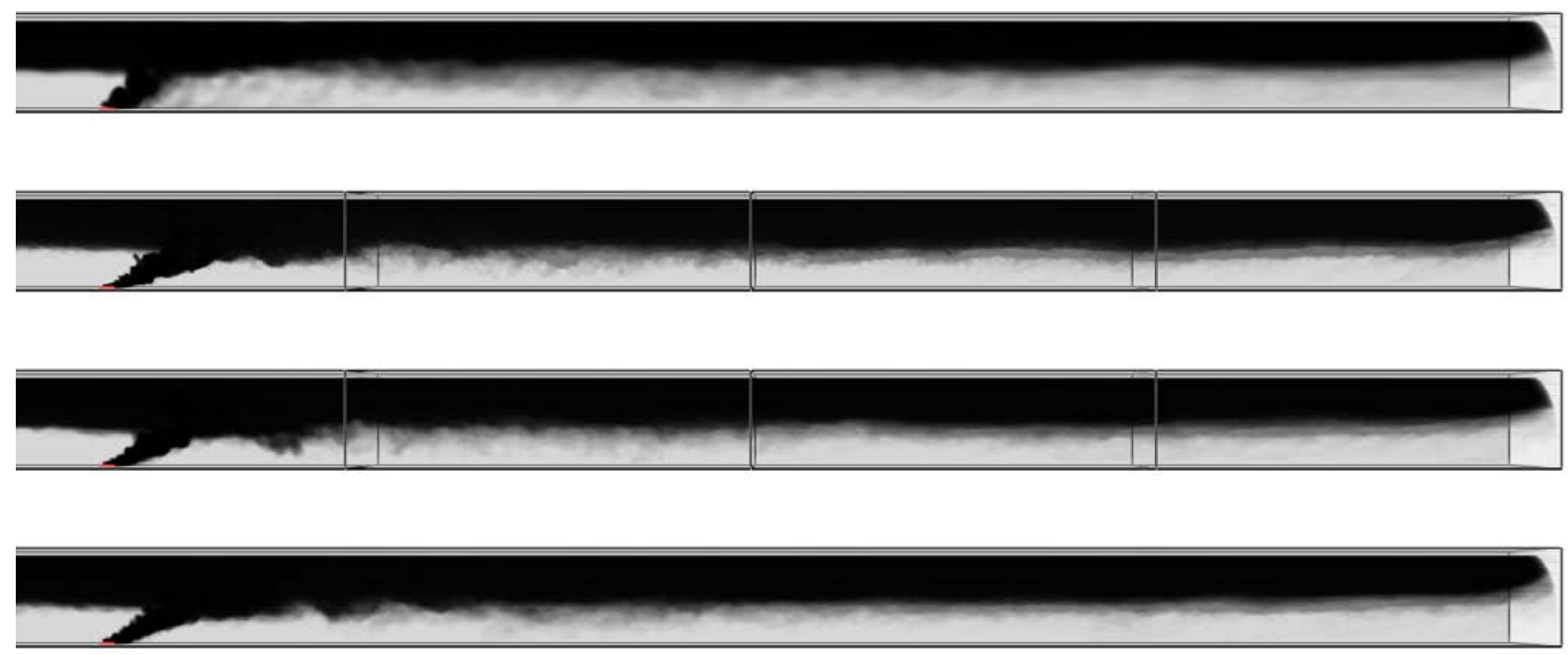

Fig 2. Fire smoke movements in the tunnel for simulations $20 \mathrm{~cm}, 5 \mathrm{~cm}-8 \mathrm{M}, 10 \mathrm{~cm}-8 \mathrm{M}$ and $10 \mathrm{~cm}$ after $200 \mathrm{~s}$.
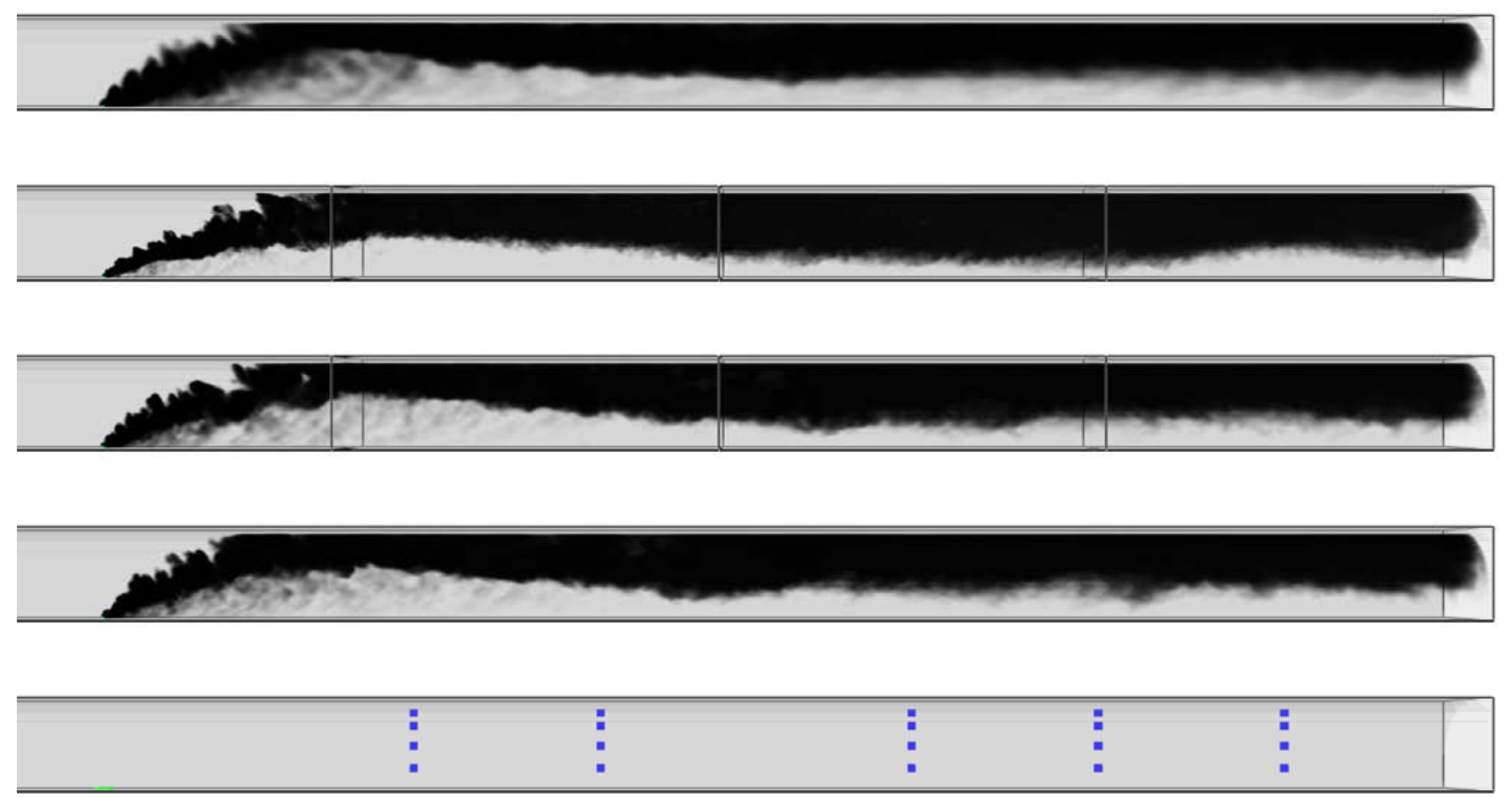

Fig 3. Aerosol movements in the tunnel for simulations $20 \mathrm{~cm}, 5 \mathrm{~cm}-8 \mathrm{M}, 10 \mathrm{~cm}-8 \mathrm{M}$ and $10 \mathrm{~cm}$ after $200 \mathrm{~s}$ and the position of detectors of optical density.

\subsection{Parallelisation of the fire smoke movement simulation}

Tab 1, column 2 shows the differences between selected couples of fire scenarios simulations. It can be seen that there are only negligible differences caused by computational domain decomposition (rows 2 and 6). For this reason, the mesh resolution is the main factor influencing simulation results and three simulations for particular three mesh resolutions are sufficient for comparison.

Sum of squared differences between 5 and $10 \mathrm{~cm}$ resolution simulations is about two times less than the sum for 10 and $20 \mathrm{~cm}$ resolutions, indicating convergence for $5 \mathrm{~cm}$ mesh resolution. Note that the contribution of the first quadruple of detectors to the final sum is dominant in all tested simulation couples. Therefore, the main loss of accuracy occurs in the fire vicinity. Dependence of simulation accuracy on mesh resolution is very weak for the tunnel section in which steady-state movement of the smoke is formed, i.e. in the area relevant for our investigation. This conclusion is supported also by Fig. 2. Perfect smoke stratification is maintained for $5 \mathrm{~cm}$ mesh resolution in the fire vicinity, while for $20 \mathrm{~cm}$ resolution sparse smoke below the smoke layer can be observed. However, in a larger 
distance, the smoke pattern is almost identical for both cases, as well as the values of optical density (see Fig. 4).

Table 1. Sum of squared differences of optical density values $\left[\mathrm{m}^{-2}\right]$ for selected simulations couples for fire and aerosol scenarios, respectively

\begin{tabular}{|c|c|c|}
\hline Simulations & Sum (fire) & $\begin{array}{c}\text { Sum } \\
\text { (aerosol) }\end{array}$ \\
\hline $20 \mathrm{~cm}-8 \mathrm{M}$ vs. $20 \mathrm{~cm}$ & 0,002 & 0,121 \\
\hline $10 \mathrm{~cm}$ vs. $20 \mathrm{~cm}$ & 0,026 & 0,029 \\
\hline $10 \mathrm{~cm}-8 \mathrm{M}$ vs. $20 \mathrm{~cm}$ & 0,030 & 0,016 \\
\hline $5 \mathrm{~cm}-8 \mathrm{M}$ vs. $20 \mathrm{~cm}$ & 0,038 & 0,049 \\
\hline $10 \mathrm{~cm}-8 \mathrm{M}$ vs. $10 \mathrm{~cm}$ & 0,001 & 0,024 \\
\hline $5 \mathrm{~cm}-8 \mathrm{M}$ vs. $10 \mathrm{~cm}$ & 0,016 & 0,087 \\
\hline $10 \mathrm{~cm}-8 \mathrm{M}$ vs. $20 \mathrm{~cm}-8 \mathrm{M}$ & 0,039 & 0,132 \\
\hline
\end{tabular}

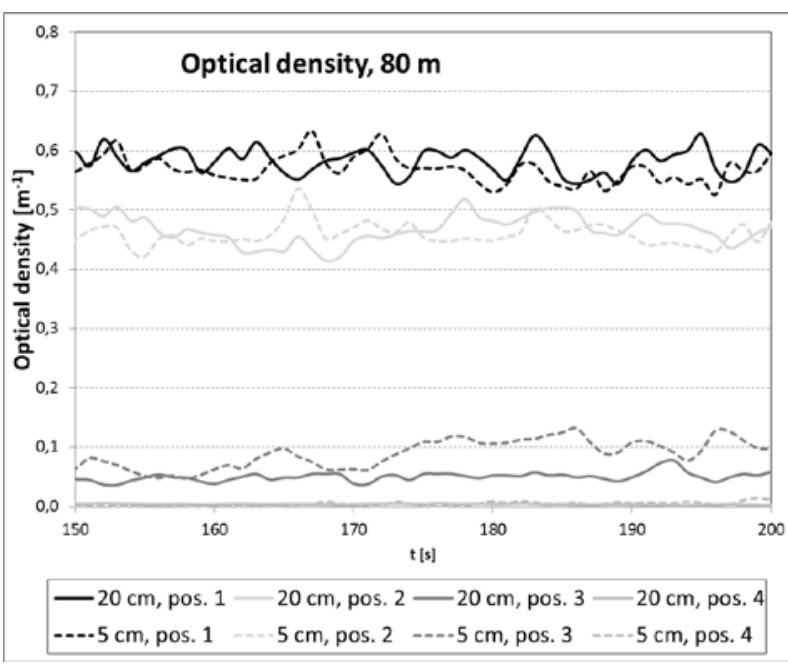

Fig. 4. Optical density of fire smoke for 5 and $20 \mathrm{~cm}$ mesh resolution measured by quadruple of detectors at $80 \mathrm{~m}$ from the fire source after steady-state conditions are formed.

\subsection{Parallelisation of the aerosol movement simulation}

Tab 1, column 3 shows the differences between selected couples of aerosol scenarios simulations. It is obvious that in contrast to the fire scenarios, the impact of domain decomposition is considerable especially for 20 $\mathrm{cm}$ mesh resolution (row 2). The combination of coarse mesh resolution and domain decomposition evidently decreases simulation accuracy. The reason of such behaviour lies probably in a numerical grid which is very coarse with respect to the extremely small HRR of a chemical reaction generating aerosol, while the same mesh resolution in the case of $1 \mathrm{MW}$ fire provides significantly more accurate description of fire dynamics. As can be expected, for $10 \mathrm{~cm}$ mesh resolution the effect is significantly weaker, although it is stronger than in the case of fire scenarios.

Sum of squared differences of optical density between 5 and $10 \mathrm{~cm}$ resolution simulations is considerable, indicating that even finer resolution than 5 $\mathrm{cm}$ would be appropriate to achieve sufficient simulation accuracy. Due to the extremely high computational requirements and wall-clock time the execution of such simulation is especially difficult. Note that in contrast to the fire smoke scenarios, there is a significant contribution of all five quadruples of detectors to the final sum. It corresponds to the noticeable differences among aerosol movements downstream of the aerosol source in scenarios with different mesh resolution (see Fig 3). However, as can be seen in Fig. 5, the 5 and 20 $\mathrm{cm}$ mesh resolutions scenarios provide reasonable estimate sufficient for practical purposes of smoke visualisation downstream of the fire.

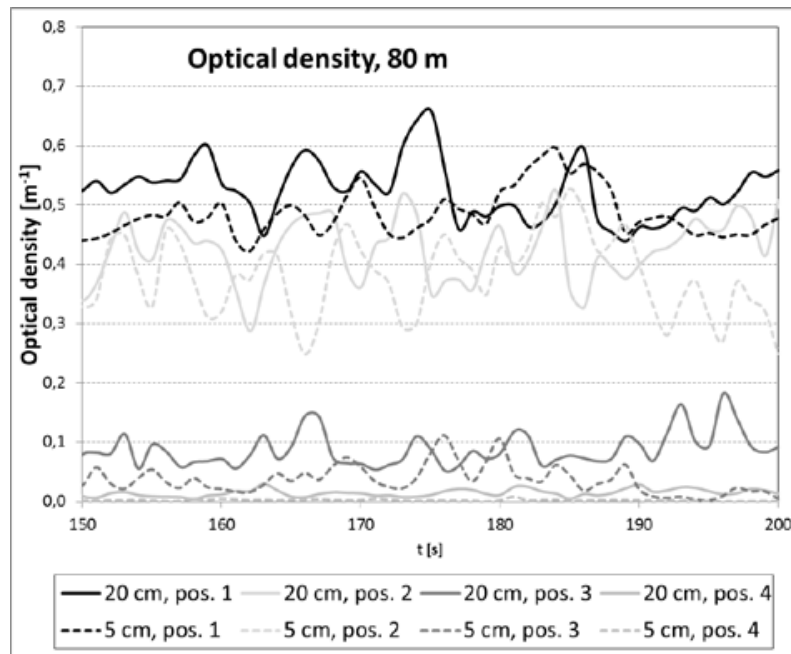

Fig. 5. Optical density of aerosol for 5 and $20 \mathrm{~cm}$ mesh resolution measured by quadruple of detectors at $80 \mathrm{~m}$ from the fire source after steady-state conditions are formed.

\subsection{Comparison of the fire and aerosol movement}

Sum of squared differences of optical density values obtained in fire and aerosol scenarios can be used as a measure of similarity of optical density profiles for both cases. Tab. 2 shows the sum for particular mesh resolution and computational domain decomposition. The contribution of particular quadruples of detectors to the sum is distinguished.

Table 2. Sum of squared differences of optical density values $\left[\mathrm{m}^{-2}\right]$ obtained in fire and aerosol scenarios for particular mesh resolution and computational domain decomposition, evaluated

for particular quadruple of detectors at specific distance, summed for detectors 1 - 5 and 3 - 5, respectively.

\begin{tabular}{|c|c|c|c|c|c|c|c|}
\hline & $25 \mathrm{~m}$ & $40 \mathrm{~m}$ & $65 \mathrm{~m}$ & $80 \mathrm{~m}$ & $95 \mathrm{~m}$ & $\begin{array}{c}\text { Sum } \\
1-5\end{array}$ & $\begin{array}{c}\text { Sum } \\
3-5\end{array}$ \\
\hline $5 \mathrm{~cm}-8 \mathrm{M}$ & 0,12 & 0,29 & 0,17 & 0,02 & 0,04 & 0,64 & 0,23 \\
\hline $10 \mathrm{~cm}$ & 0,21 & 0,40 & 0,13 & 0,01 & 0,15 & 0,89 & 0,28 \\
\hline $10 \mathrm{~cm}-8 \mathrm{M}$ & 0,21 & 0,36 & 0,18 & 0,01 & 0,10 & 0,87 & 0,30 \\
\hline $20 \mathrm{~cm}$ & 0,17 & 0,38 & 0,13 & 0,01 & 0,07 & 0,76 & 0,21 \\
\hline $20 \mathrm{~cm}-8 \mathrm{M}$ & 0,11 & 0,30 & 0,22 & 0,04 & 0,01 & 0,67 & 0,27 \\
\hline
\end{tabular}

It can be seen that the simulation settings have only slight impact on the assessment of similarity between optical density profiles of fire and aerosol scenario. For 
every mesh resolution and computational domain decomposition there are significant differences measured by the first and second quadruple of detectors, while the contribution of the latter quadruple to the sum is dominant. The contribution of other three quadruples of detectors are noticeably lower, which means that after steady-state conditions are formed, optical density profiles for both scenarios are very similar regardless of mesh resolution and the way of domain decomposition.

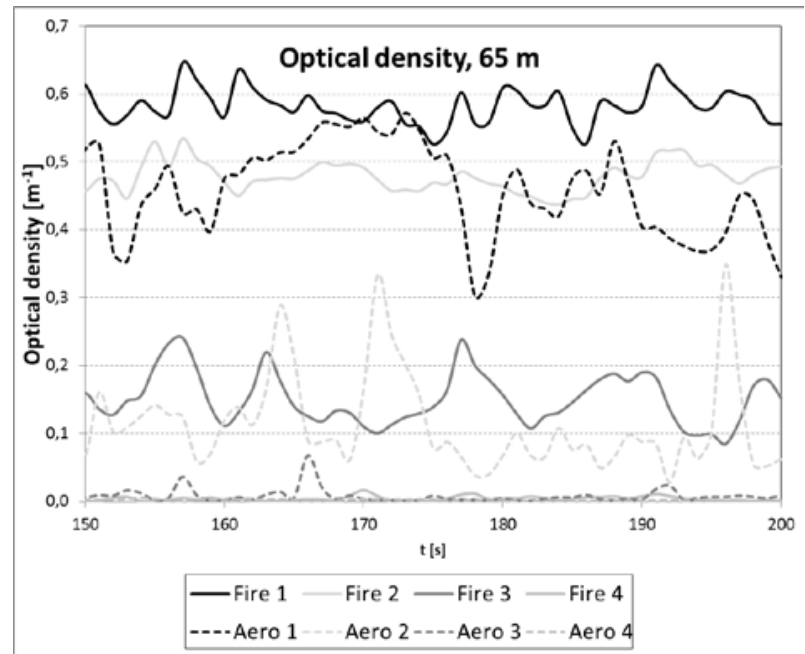

Fig. 6. Optical density for the fire and the corresponding aerosol scenario measured by quadruple of detectors at $65 \mathrm{~m}$ from the fire/aerosol source after steady-state conditions are formed ( $5 \mathrm{~cm}$ mesh resolution).

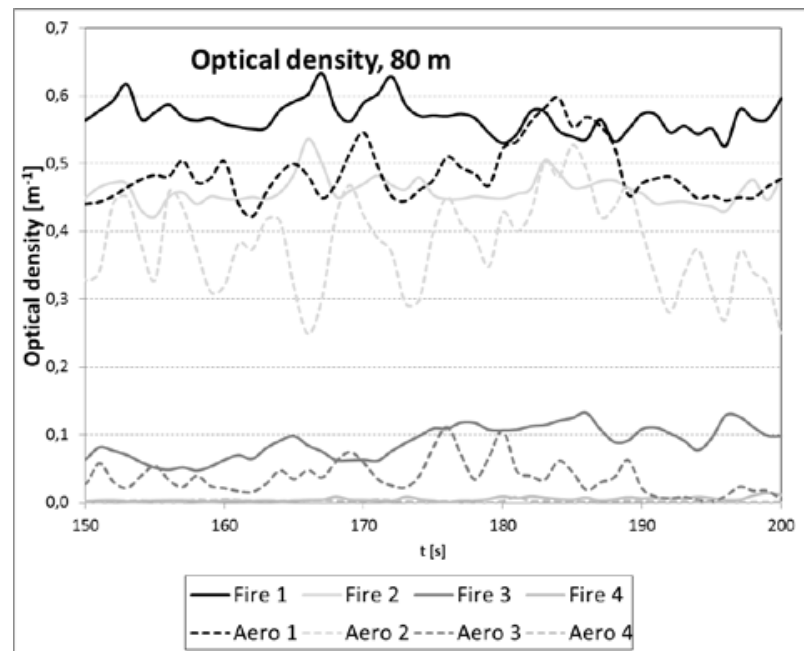

Fig. 7. Optical density for the fire and the corresponding aerosol scenario measured by quadruple of detectors at $80 \mathrm{~m}$ from the fire/aerosol source after steady-state conditions are formed (5 $\mathrm{cm}$ mesh resolution).

Noticeable differences can be observed at the third quadruple of detectors located at $65 \mathrm{~m}$ at which the optical density profile is not fully stabilized (see also Fig. 6). The best highest similarity is obtained at $80 \mathrm{~m}$ distance, where the both profiles are almost identical (see Figures 7 and 8). At $95 \mathrm{~m}$, the similarity becomes slightly worse, probably due to the flow of cold air from the tunnel portal occurring in the fire scenarios, which is not present in the aerosol scenarios. However, this effect is not relevant for our study, as the ventilation tests deal mainly with the smoke movement inside the tunnel and slight differences between both scenarios near the tunnel portals are not important. It can be assumed that in a longer tunnel than is our testing tunnel reasonable similarity would be achieved in a relatively long section of the tunnel past $70 \mathrm{~m}$ away from the fire/aerosol source.

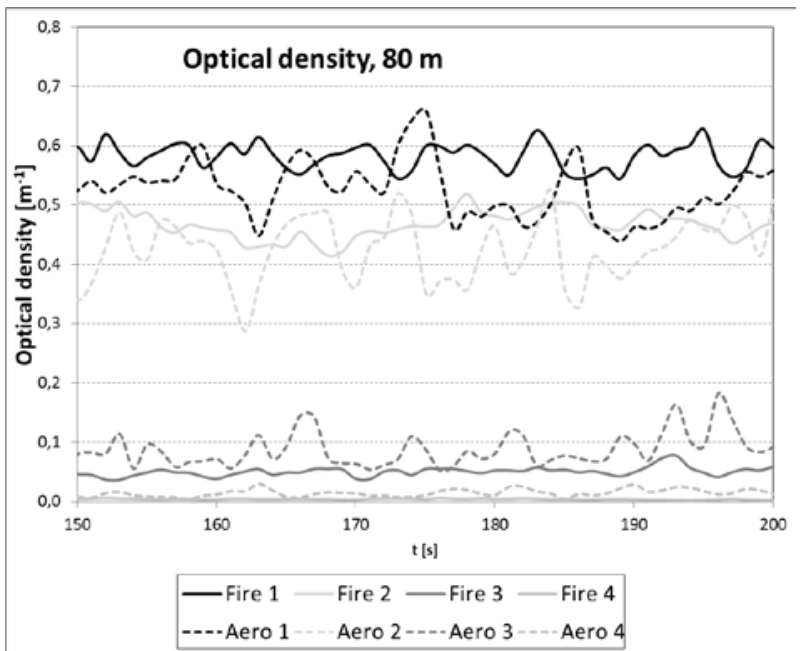

Fig. 8. Optical density for the fire and the corresponding aerosol scenario measured by quadruple of detectors at $80 \mathrm{~m}$ from the fire/aerosol source after steady-state conditions are formed (20 cm mesh resolution).

Note that the similarity between both scenarios must finally disappear as the smoke stratification is maintained longer in the fire smoke scenario due to the higher HRR.

\section{Conclusions}

Series of simulations investigating optical density of fire smoke and testing aerosol used for ventilation tests in road tunnels were performed using the Fire Dynamics Simulator system. The accuracy of simulations with 5, 10 and $20 \mathrm{~cm}$ mesh resolution for sequential computation and for parallel computation using $8 \mathrm{MPI}$ processes was evaluated. The results indicate significant similarity between optical density profiles of the aerosol and fire smoke for the steady-state movement formed downstream of the fire/aerosol source. The mesh resolution and the way of computational domain decomposition do not have significant impact on this similarity. In case of fire smoke, the influence of domain decomposition is negligible, while the impact of mesh resolution is slightly more pronounced. More significant differences could be observed among particular aerosol scenarios due to the numerical grid which is relatively coarse with respect to the small HRR of a chemical reaction generating aerosol. Nevertheless, the results indicate that the testing aerosol is suitable to represent the steady-state movement of fire smoke downstream of the smoke source in full-scale road tunnel ventilation tests. 


\section{Acknowledgements}

The authors would like to thank P. Schmidt, B. Spisakova (National Motorway Company) for information about technical specifications of road tunnels and M. Dobrucky, J. Astalos and V. Sipkova for technical support for SIVVP HPC cluster computing. This paper was partially supported by the Slovak Science Foundation (project No. VEGA 2/0165/17) and the Slovak Research and Development Agency (project No. APVV-15-0340).

\section{References}

[1] TP 12/2011 Road tunnels ventilation (in Slovak), (Ministry of Transport and Construction of the Slovak Republic, 2011)

[2] Guideline - Road tunnels ventilation (in Czech), (Ministry of Transport of the Czech Republic, 2013)

[3] H. Ingason, Y.Z. Li, A. Lönnermark, Tunnel fire dynamics (Springer-Verlag, New York, 2015, p. 321)

[4] P. Weisenpacher, J. Glasa J, L. Valasek, Proceedings of 10th Mediterranean Combustion Symposium, Naples, Italy (2017)

[5] http://ndsas12.cmsromboid.sk/prehladtunelov/44346s

[6] http://www.sta-itaaites.sk/index.php?page=tunely_vo_vystavbe [7] M. Bebcak, P. Bebcak, Proceedings of Fire Safety of Tunnels, Hradec nad Moravici, Czech Republic (2013)

[8] P. Weisenpacher, J. Glasa, L. Valasek, Journal of Physics: Conference Series (in press).

[9] K. McGrattan, S. Hostikka, R. McDermott, J. Floyd, C. Weinschenk, K. Overholt, Fire Dynamics Simulator, Technical Reference Guide (National Institute of Standards and Technology, Gaithersburg, Maryland, USA, and VTT Technical Research Centre of Finland, Espoo, Finland, sixth edition, 2017)

[10] K. McGrattan, S. Hostikka, R. McDermott, J. Floyd, C. Weinschenk, K. Overholt, Fire Dynamics Simulator, User's Guide (National Institute of Standards and Technology, Gaithersburg, Maryland, USA, and VTT Technical Research Centre of Finland, Espoo, Finland, sixth edition, 2017)

[11] P. Weisenpacher, J. Glasa, L. Halada, L. Valasek, V. Sipkova, Comput. Inform. 331237 (2014)

[12] SIVVP - Slovak Infrastructure for High Performance Computing. Available from: http://www.sivvp.sk/, http://hpc.ui.savba.sk/

[13] P. Danisovic, J. Sramek, M Hodon, M. Hudik MATEC Web of Conferences 11700035 (2017)

[14] M Sirilla, P. Schmidt (in Slovak) Inzinierske stavby 656 (2017) 